\title{
Spurious-Free Dynamic Range of a Uniform Quantizer
}

\author{
Mark S. Oude Alink, André B. J. Kokkeler, Eric A. M. Klumperink, Senior Member, IEEE, \\ Kenneth C. Rovers, Gerard J. M. Smit, and Bram Nauta, Fellow, IEEE
}

\begin{abstract}
Quantization plays an important role in many systems where analog-to-digital conversion and/or digital-to-analog conversion take place. If the quantization error is correlated with the input signal, then the spectrum of the quantization error will contain spurious peaks. Although analytical formulas describing this effect exist, numerical evaluation can take much effort. This brief provides approximations for the spurious-free dynamic range (SFDR) of a uniform quantizer with a single sinusoidal input, with and without additive Gaussian noise. It is shown that the SFDR increases by approximately $8 \mathrm{~dB} / \mathrm{bit}$, in case there is no noise. Generalizing this result to multitone inputs results in an additional $2 \mathrm{~dB} / \mathrm{bit}$ per additional tone. Additive Gaussian noise decorrelates the sinusoid(s) and the quantization error, which results in a dramatic increase in SFDR.
\end{abstract}

Index Terms-Analog-to-digital conversion, digital-to-analog conversion, harmonic distortion, intermodulation distortion, polynomial approximation, quantization, quantization noise, spurious-free dynamic range (SFDR).

\section{INTRODUCTION}

Q UANTIZATION is an important step in analog-to-digital conversion. It limits a signal to a finite set of discrete values that, in general, will not exactly represent the original signal. In most cases, the quantization levels are uniformly spaced at a distance known as the LSB.

The quantization error is defined as the difference between the output and input signals of the quantizer. It is usually assumed that the error is uniformly distributed between $-1 / 2$ LSB and 1/2 LSB, with an equal amount of power for each frequency, i.e., it is modeled as white noise and is independent of (and, hence, uncorrelated with) the input signal. The total power of this quantization noise can then be calculated to be $\mathrm{LSB}^{2} / 12$.

Applying the preceding assumptions and taking a full-scale sinusoid as the input signal, it can be shown that the SNR at the output is equal to

$$
\mathrm{SNR}=6.02 b+1.76[\mathrm{~dB}]
$$

where $b$ denotes the number of bits.

Manuscript received October 4, 2008; revised December 24, 2008. Current version published June 17, 2009. This paper was recommended by Associate Editor H.-S. Chen.

M. S. Oude Alink is with the Computer Architecture for Embedded Systems (CAES) Group and the IC-Design Group, Centre for Telematics and Information Technology (CTIT), University of Twente, 7500 AE Enschede, The Netherlands (e-mail: m.s.oudealink@utwente.nl).

A. B. J. Kokkeler, K. C. Rovers, and G. J. M. Smit are with the CAES Group, CTIT, University of Twente, 7500 AE Enschede, The Netherlands.

E. A. M. Klumperink and B. Nauta are with the IC-Design Group, CTIT, University of Twente, 7500 AE Enschede, The Netherlands.

Digital Object Identifier 10.1109/TCSII.2009.2020929
However, in reality, the quantization error can (partly) be correlated with the input signal. The result is that the correlated part of the quantization error is not translated into a white noise floor, but into distortion of the input signal. Nevertheless, the total power of the distortion components is still relatively well approximated by (1), particularly for a larger number of quantization levels [1], [2].

For a sine wave input without noise, uniform quantization results in pure harmonic distortion. The difference in power between the desired signal and the most powerful distortion component is termed as the spurious-free dynamic range (SFDR). The SFDR is an important specification for spectrum analyzers, because a user cannot distinguish between distortion components and true input signals. It is also relevant for receivers, because the SFDR may limit receiver sensitivity [1].

Increasing the resolution of the quantizer increases the SFDR, but the resolution of analog-to-digital converters (ADCs) is limited by the required sampling rate and the maximum allowable power consumption [3]. Analytical formulas for distortion components are available, but they are difficult to evaluate and do not easily translate to the SFDR. Therefore, system designers would benefit from simple design equations for the SFDR to allow for the exploration of the design space and optimization between resolution, SNR, and SFDR.

Quantization also plays a role in digital-to-analog converters (DACs). An important type of DAC is the zero-order-hold (ZOH) DAC, which retains the sample value until the following sample. For a uniformly quantized digital signal, the result is a uniformly quantized analog signal. Therefore, the analyses to be presented apply equally well to ZOH DACs.

In this brief, we will derive approximations for the SFDR using a numerical evaluation of the analytical formulas, which leads to simple closed-form expressions. We will also look at quantization in the presence of noise and briefly touch upon the quantization of multiple tones. In both cases, the correlation is reduced, which results in an increase of the SFDR. Nonuniform quantization and clipping also introduce distortion, but that is not considered in this brief.

It is vital to note that sampling is not considered, because it is orthogonal to quantization. When no sampling takes place, the spectrum of a quantized signal may contain components at any frequency. When sampled, these components will alias if their frequencies are higher than the Nyquist frequency.

\section{QuAnTIZATION OF A Sinusoid}

The multilevel quantization of a sinusoid that is free of noise has mathematically been investigated by Blachman [4]. It was shown that for a midriser quantizer (i.e., a quantizer with a 


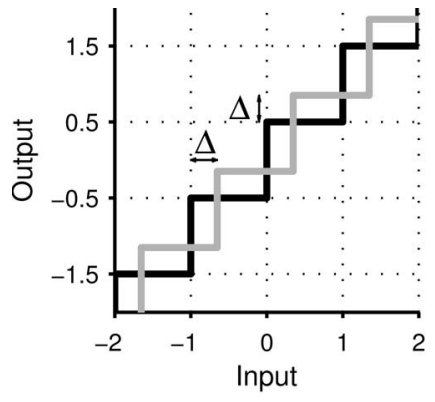

(a)

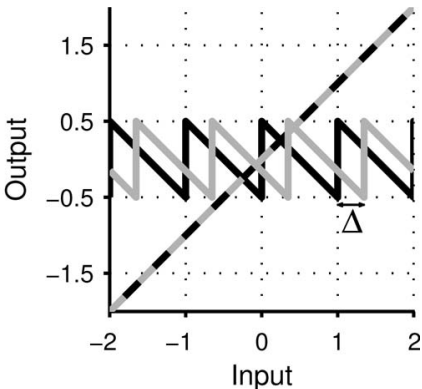

(b)
Fig. 1. The quantization staircase of (a) can be decomposed into a straight line and a repetitive quantization error as shown in (b). The black line represents $\Delta=0$, which corresponds to a midriser quantizer.

threshold exactly at 0 ), only odd-order harmonics are produced due to the odd-symmetric nature of the quantization staircase. To simplify the calculations, all the amplitudes in this brief are expressed in LSB, and one LSB is normalized to 1. The resulting output signal then is equal to [4]

$$
A_{p}=\delta_{p, 1} A+\sum_{m=1}^{\infty} \frac{2}{m \pi} J_{p}(2 m \pi A)
$$

where $A_{p}$ is the output amplitude of the $p$ th harmonic, $\delta_{i, j}$ is the Kronecker delta function, $A$ is the input amplitude, and $J_{p}$ is the $p$ th-order Bessel function of the first kind.

Using the quantization staircase $q(x)$, as shown in Fig. 1, (2) can be generalized to uniform quantizers by expressing it as a linear transfer plus the quantization error. This quantization error is periodic with a period of $1 \mathrm{LSB}$. As a result, $q(x)$ can be written as the sum of $x$ and the Fourier series of the quantization error as

$$
q(x)=x+\sum_{m=1}^{\infty} a_{m} \sin (2 \pi m x)-\sum_{m=1}^{\infty} b_{m} \cos (2 \pi m x)
$$

where the coefficients can be found by straightforward calculation, which results in

$$
a_{m}=\frac{2 \cos ^{2}(\Delta \pi m)-1}{\pi m} \quad b_{m}=\frac{\sin (2 \pi \Delta m)}{\pi m}
$$

where $\Delta$ is the offset in LSB. Using the same method as Blachman [4], we then find

$$
A_{p}= \begin{cases}\left(2-\delta_{p, 0}\right) \sum_{m=1}^{\infty} b_{m} J_{p}(2 \pi m A), & \text { for } p \text { even } \\ 2 \sum_{m=1}^{\infty} a_{m} J_{p}(2 \pi m A)+\delta_{p, 1} A, & \text { for } p \text { odd }\end{cases}
$$

which reduces to (2) for $\Delta=0$.

Because $A$ is expressed in LSB, the number of quantization levels $n$ directly depends on the amplitude $A$ of the sinusoid. Hence, $n$ does not need to be a power of two, which is important because in practical situations sinusoids are not always full scale.

Fig. 2 shows the lower part of the spectrum of a full-scale sinusoid quantized with 8 bits $(A=128)$, which is obtained by numerical evaluation of (2) and by simulation (both in MATLAB). Note again that sampling is not considered, which

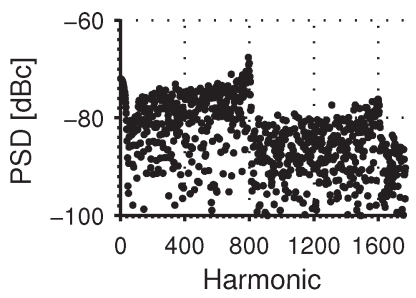

(a)

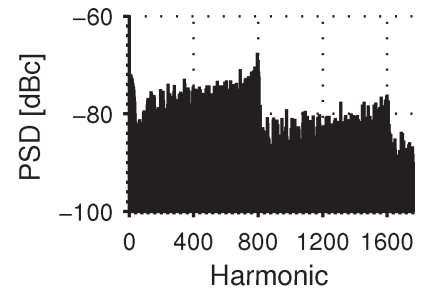

(b)
Fig. 2. Low end of the spectrum of a full-scale sinusoid after 8-bit quantization (a) using (2) and (b) using simulation.

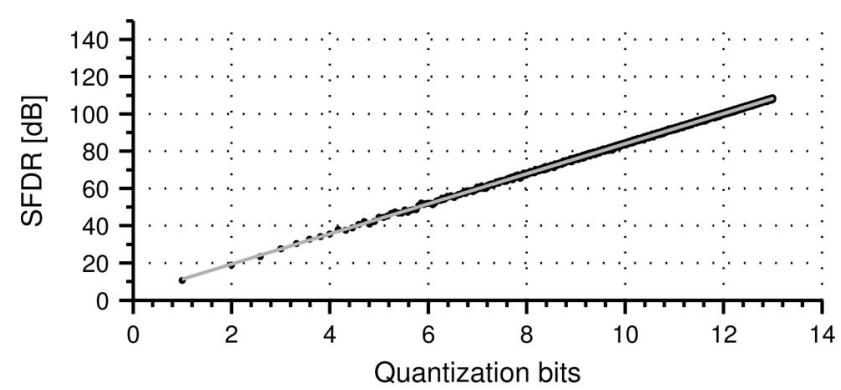

(a)

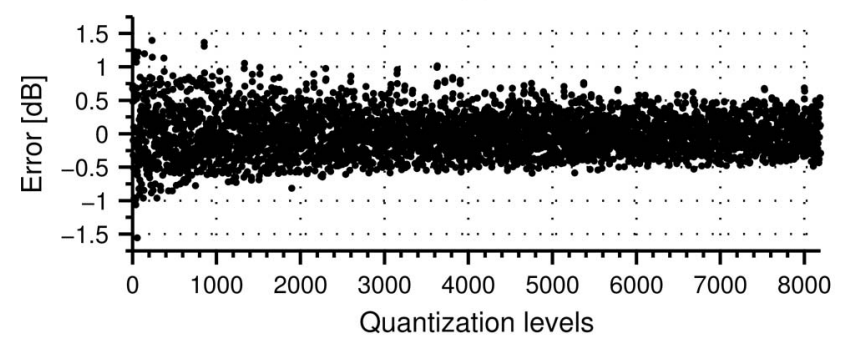

(b)

Fig. 3. (a) SFDR for a full-scale sinusoid as a function of the number of quantization levels for a midriser quantizer (points) and linear fit (line). (b) Error of the linear fit.

in simulations is approximated by using a sampling rate that is high enough to make the aliasing effects negligible. For the harmonic $p$ with the highest power, $p$ is equal to 795 , which is close to $256 \pi$, as derived by Blachman [4]. Numerical evaluation (not shown here) indicates that the approximation of the strongest harmonic being located roughly at $2 \pi A$ is only valid for at least 20 quantization levels. In other cases, the third harmonic is the strongest.

Pan and Abidi [1] simulated the effect of midriser quantization of a sinusoid. They constructed two linear fits for the power of the highest harmonic as a function of the number of bits, both with a slope of $9 \mathrm{~dB} / \mathrm{bit}$, but with different offsets. Although these fits were intuitively explained, it can be seen from their simulation results [1, Fig. 3] that the true slope is somewhat less than $9 \mathrm{~dB} / \mathrm{bit}$.

We determined the SFDR through numerical evaluation (and verified by simulation) for a full-scale sinusoid with $\Delta=0$ for all even values of $n$ (to keep symmetry around zero) up to 13 bits, as shown in Fig. 3. A linear fit (also shown in Fig. 3) of these points results in

$$
\mathrm{SFDR}_{\sigma_{N=0}}=8.07 b+3.29[\mathrm{~dB}]
$$

where $b=\log _{2} n$. The subscript $\sigma_{N}=0$ is added to denote the absence of noise. Because $n$ is not necessarily a power of 2 , 


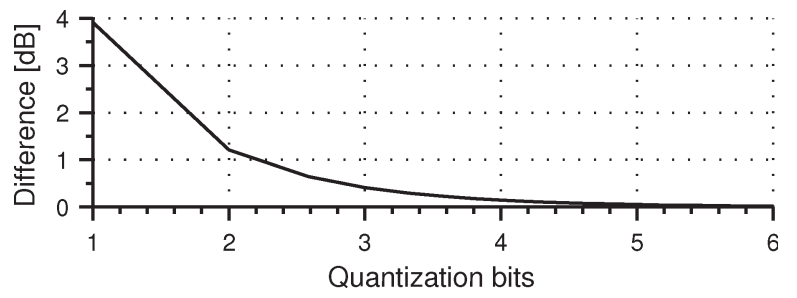

Fig. 4. Difference in decibels between the SFDR related to the input power and the fundamental output power.

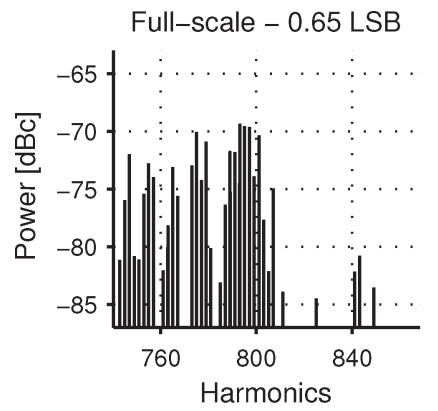

(a)

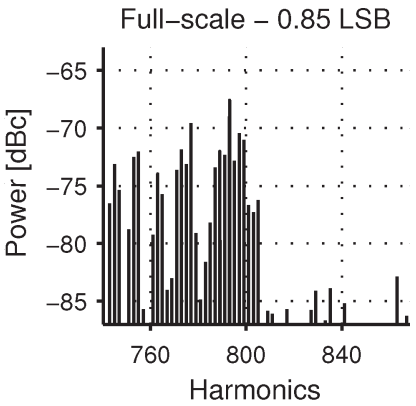

(b)
Fig. 5. Zoom-in on the spectrum for an 8-bit quantization of a sinusoid with full-scale amplitude (a) minus 0.65 LSB and (b) minus 0.85 LSB. The SFDR is 69.85 and $67.60 \mathrm{~dB}$, respectively. The "holes" in the spectra contain values below the range shown.

$b$ is not necessarily an integer. This approximation has a mean absolute error of $0.25 \mathrm{~dB}$ and a standard deviation of $0.31 \mathrm{~dB}$, with a maximum error of $1.56 \mathrm{~dB}$ occurring for 58 quantization levels. Numerical evaluation showed this approximation to hold for up to at least 25 bits.

There is one ambiguity in the definition of the SFDR that needs to be clarified. In the case of only a few quantization levels, the first harmonic (or fundamental component) is significantly lower in amplitude at the output than at the input. For example, in the case of 1-bit quantization of a full-scale sinusoid, the power of the output fundamental is $3.9 \mathrm{~dB}$ lower than the input power. One could define the SFDR with respect to the input amplitude (which is the usual definition) or with respect to the output amplitude. The difference is shown in Fig. 4. It can be seen that for 4 bits or more, the difference is negligible. The linear fit described in (6) uses the SFDR with respect to the output fundamental, which seems to be in accordance with the choice made by Pan and Abidi [1].

For an arbitrary value of $\Delta$, both even- and odd-order harmonics are present, which means the distortion power is distributed over more distortion components. Indeed, numerical evaluation shows that when $a_{m}$ in (5) is set equal to $b_{m}$ for $m=1$ (for reasons to be discussed in the derivation), which is the case for $\Delta=1 / 8$ and $\Delta=5 / 8$, the SFDR increases by roughly $3 \mathrm{~dB}$ as compared with (6).

In practice, the amplitude will never be exactly full scale. Numerical evaluation shows that the SFDR randomly changes with a magnitude similar to the error shown in Fig. 3 if the amplitude is somewhere in the range between full scale and full scale minus one LSB. An example of this change is shown in Fig. 5. This suggests that approximations to the theoretical value may deviate by 1 or $2 \mathrm{~dB}$ without compromising practical relevance.

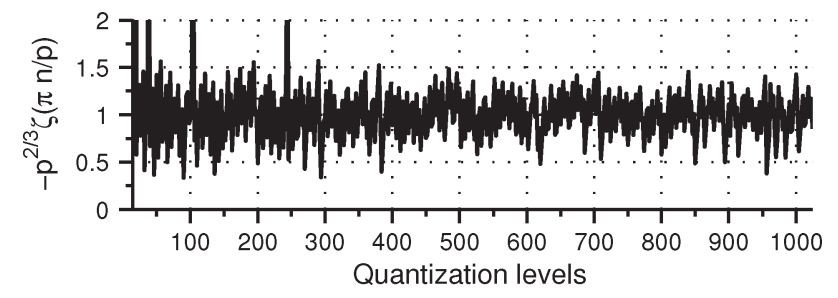

Fig. 6. Numerical evaluation of $\hat{p}^{2 / 3} \zeta(\pi n / \hat{p})$ for a midriser quantizer with $n$ ranging from 20 to 1024 .

\section{A. Derivation of $8 \mathrm{~dB} / \mathrm{bit}$}

Blachman [4] showed that the harmonics for $p \ll 2 \pi A$ decrease by $3.01 \mathrm{~dB} / \mathrm{bit}$. In his case, the LSB remains 1 . In practice, the maximum amplitude is often given, and one would like to see what happens if the resolution is increased. Therefore, an extra bit means that the LSB is divided by two. This corresponds to another $6.02 \mathrm{~dB} / \mathrm{bit}$ decrease, so the net result is a decrease of $9.03 \mathrm{~dB} / \mathrm{bit}$. This was also the conclusion drawn by Pan and Abidi [1].

However, we found that the harmonics around $p \approx 2 \pi A$ decrease by only about $2 \mathrm{~dB} /$ bit (leaving the LSB at 1 ). We will derive this from the exact analytic formula [see (5)] by using approximations to the Bessel function and showing that only the first term in the summation contributes to the trend.

As suggested in [4], the Bessel functions can be approximated by Airy functions in the region where $p \approx 2 \pi A[5$, p. 366, eq. (9.3.6)]

$$
J_{p}(p z) \approx\left(\frac{4 \zeta(z)}{1-z^{2}}\right)^{\frac{1}{4}} \frac{\mathrm{Ai}\left(p^{\frac{2}{3}} \zeta(z)\right)}{p^{\frac{1}{3}}}
$$

where

$$
\zeta(z)=-\left(\frac{3}{2} \sqrt{z^{2}-1}-\frac{3}{2} \arccos \frac{1}{z}\right)^{\frac{2}{3}} .
$$

With the strongest harmonic $\hat{p}$ located at $\hat{p} \approx 2 \pi A$, (2) in combination with (7) yields

$$
A_{\hat{p}} \approx 2 \sum_{m=1}^{\infty} c_{m}\left(\frac{4 \zeta(z)}{1-z^{2}}\right)^{\frac{1}{4}} \frac{\mathrm{Ai}\left(\hat{p}^{\frac{2}{3}} \zeta(z)\right)}{\hat{p}^{\frac{1}{3}}}
$$

where $c_{m}=a_{m}$ if $\hat{p}$ is odd, $c_{m}=b_{m}$ if $\hat{p}$ is even, and $z=2 \pi m A / \hat{p}$.

The cases $m=1$ and $m>1$ in (9) are considered separately. It will turn out that only the $m=1$ term in the summation contributes to the trend, whereas the other terms merely act as "random" deviations from this trend.

Numerical analysis shows that $\hat{p}$ is always slightly smaller than $2 \pi A$, but tends to approach $2 \pi A$ for a larger $A$. For $m=1$, we numerically find $z \in(1 ; 1.1)$. In this region, the factor $4 \zeta(z) /\left(1-z^{2}\right)$ remains virtually constant.

The parameter $\hat{p}^{2 / 3} \zeta(2 \pi A / \hat{p})$ of the Airy function was numerically evaluated for a midriser quantizer quantizing a full-scale sinusoid for $n=20$ to $n=1024$ and is shown in Fig. 6. Clearly, this parameter stays roughly -1 , which indicates that $\operatorname{Ai}\left(\hat{p}^{2 / 3} \zeta(2 \pi A / \hat{p})\right)$ can also be considered to be a constant. 


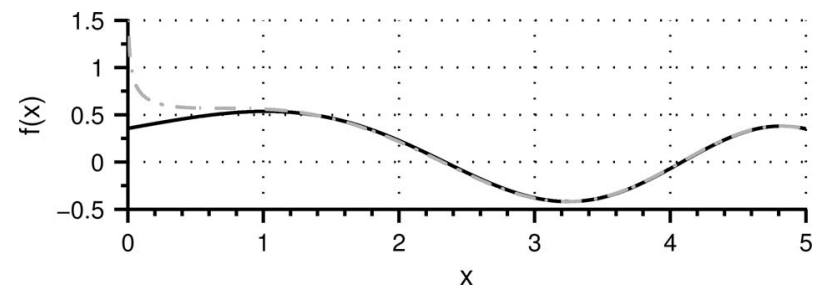

Fig. 7. Airy function $\operatorname{Ai}(-x)$ (solid line) and the approximation of (11) (dashed line).

Removing the constants, we, therefore, obtain for the first term in the summation of (9)

$$
A_{\hat{p}}(m=1) \propto \frac{1}{\hat{p}^{\frac{1}{3}}}
$$

which corresponds to a $\hat{p}^{-(2 / 3)}$ dependency in the power spectrum, which is equivalent to a decrease of $2.01 \mathrm{~dB} / \mathrm{bit}$.

For $m \geq 2$, we approximate the Airy function using [5, p. 449 , eq. (10.4.83)]

$$
\operatorname{Ai}(-x) \approx \frac{\sin \left(\frac{2}{3} x^{\frac{3}{2}}+\frac{\pi}{4}\right)}{\sqrt{\pi} x^{\frac{1}{4}}}
$$

for $x \gg 1$. Fig. 7 shows that this approximation is quite accurate for $x>1.2$ (error less than 0.013).

Substituting this and the approximation $z=2 \pi m A / \hat{p} \approx m$ into (7) and simplifying, we obtain

$$
J_{p}(p z) \approx J_{p}(p m) \approx\left(\frac{4}{m^{2}-1}\right)^{\frac{1}{4}} \frac{\sin \left(-\frac{2}{3} p \zeta(m)^{\frac{3}{2}}+\frac{\pi}{4}\right)}{\sqrt{\pi} p^{\frac{1}{2}}} .
$$

There seems to be no relation between the number of quantization levels and the phase of the sinusoid in (12). Hence, the sine term can be considered as a random variable that takes values between -1 and 1 with an expectation of 0 .

In conclusion, only the first term in the summation of (9) is important for the overall trend, whereas the other terms provide more or less random deviations. This randomness explains the erratic behavior around the trend of the SFDR shown in Fig. 3. Combining the $2.01 \mathrm{~dB} / \mathrm{bit}$ for the $m=1$ term and the $6.02 \mathrm{~dB} / \mathrm{bit}$ from halving the amplitude of the quantization error, a trend of $8.03-\mathrm{dB} /$ bit increase in SFDR is expected, which is very close to the $8.07 \mathrm{~dB} /$ bit obtained from numerical evaluation.

\section{Quantization of a Sinusoid With Noise}

Adding noise to the input signal decorrelates the input signal with the quantization error [6] and can be regarded as a form of dithering [2]. The effect only depends on the univariate probability density function (pdf) of the noise and not on its spectrum [4]. The resulting signal frequency response is the product of the quantization error, as given in (2), and the Fourier transform of the pdf of the noise, e.g., as in [4, eq. (35)].

Because the thermal noise has a Gaussian pdf and is often the most important noise contribution, we will study the effect of this noise on the SFDR in more detail. The results apply equally well to flicker noise if it is assumed that this noise has a Gaussian pdf. Any frequency component below $1 / T$, where

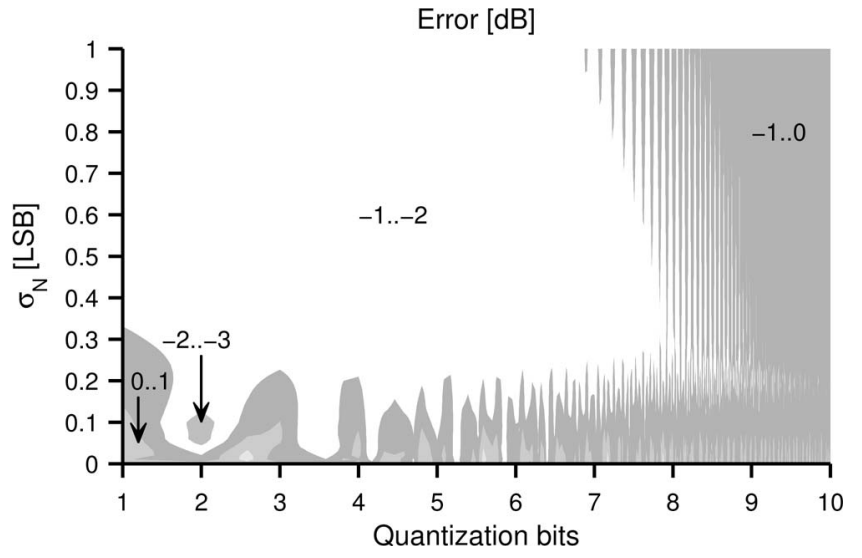

Fig. 8. Contour plot of the error made in decibels by the approximation given in (14) as compared with (13).

$T$ is the observation time, will constitute an apparent constant offset [7], which can be represented by $\Delta$ in (5). The exact resulting spectrum is found to be [4], [6], [8]

$$
A_{p}=\delta_{p, 1} A+\sum_{m=1}^{\infty} c_{m} J_{p}(2 m \pi A) e^{-2 \pi^{2} \sigma_{N}^{2} m^{2}}
$$

where $\sigma_{N}$ is the standard deviation of the noise in LSB, and $p$ denotes the $p$ th harmonic of the input frequency. Note that $p$ is completely unrelated to the noise frequencies.

Using the previously derived result that only $m=1$ contributes to the trend of the SFDR as a function of the number of quantization levels, it can be observed that the increase in SFDR (in decibels) quadratically depends on the noise level in LSB. Given as an equation, we have

$$
\begin{aligned}
\operatorname{SFDR}_{\sigma_{N}} & =\operatorname{SFDR}_{\sigma_{N=0}}+20 \log _{10} e^{-2 \pi^{2} \sigma_{N}^{2}} \\
& =8.07 b+3.29+171.5 \sigma_{N}^{2}[\mathrm{~dB}] .
\end{aligned}
$$

The result is that adding more noise increases the SFDR, which may look counterintuitive. The SFDR of, e.g., a spectrum analyzer, also takes the noise level into account, because the noise in each "frequency bin" is integrated over the (effective) noise bandwidth. For a quantizer, the concept of a resolution bandwidth is not applicable, and hence, only spurious peaks are considered.

Fig. 8 shows a contour plot of the error (in decibels) between the approximated [using (14)] and the calculated [using (13)] SFDR as a function of the two important parameters: 1) the number of quantization levels and 2) the amount of noise. The contour plot is generated by evaluating (13) and (14) for all combinations of an even number of quantization levels and $\sigma_{N}$ in steps of $0.01 \mathrm{LSB}$. Because $\exp \left(-2 \pi^{2} \sigma_{N}^{2} m^{2}\right)$ in (13) is a smooth function of $\sigma_{N}$, no smaller steps in $\sigma_{N}$ are required. A negative error means the approximation underestimates the SFDR given by theory. Clearly, the approximation is quite close over the whole range of values shown. The difference of less than $2 \mathrm{~dB}$ in virtually all cases bears no practical relevance, as discussed in Section II.

Because the instantaneous amplitude of the noise can assume any value, practical quantizers will sometimes clip, which results in (additional) distortion of the spectrum. Gaussian noise has an amplitude of less than $3 \sigma$ for $99.87 \%$ of the time. 
Simulations show that if the amplitude is full scale minus $3 \sigma$, then the clipping effects have no discernable influence on the spectrum.

\section{Multitone Quantization}

Using the derivation of the $8-\mathrm{dB} /$ bit trend for the quantization of a single sinusoid, it turns out to be quite straightforward to generalize it for an arbitrary number of sinusoids. Suppose $N$ sinusoids of frequency $f_{i}$ and amplitude $A_{i}$ are present at the input, where $i$ ranges from 1 to $N$. The output will contain peaks at frequencies $\sum_{i} p_{i} f_{i}$, with $\sum_{i} p_{i}>0$ (note that any individual $p_{i}$ can be negative), the amplitude of which is denoted by $A_{p_{1}, \ldots, p_{N}}$. This amplitude can be derived using the identities [4]

$$
e^{j z \sin (\theta)}=\sum_{p=-\infty}^{\infty} J_{p}(z) e^{j p \theta}, \quad J_{-p}(z)=(-1)^{p} J_{p}(z)
$$

resulting in

$$
\begin{aligned}
A_{p_{1}, \ldots, p_{N}}=\sum_{i=1}^{N}\left(\delta_{p_{i}, 1} A_{i} \prod_{j \neq i} \delta_{p_{j}, 0}\right) \\
+\sum_{m=1}^{\infty}\left(c_{m} \prod_{i=1}^{N} J_{p_{i}}\left(2 \pi m A_{i}\right)\right) .
\end{aligned}
$$

If some of the input frequencies are commensurate, i.e., $\exists k, l_{j} \in \mathbb{Z}$ such that $k f_{i}=\sum_{j \neq i} l_{j} f_{j}$, then multiple amplitudes $A_{p_{1}, \ldots, p_{N}}$ will occupy the same frequency and, therefore, need to be added while taking into account their respective phase relations to obtain the total amplitude at that specific frequency. This results in a much more complicated expression for the general case and is, therefore, not discussed here.

In the derivation of the trend in SFDR for a single sinusoid, we found a $p^{-(1 / 3)}$ amplitude dependence if the LSB was kept equal to 1 . Since the summation now contains the product of $N$ of these Bessel functions, we find a $p^{-(N / 3)}$ amplitude dependency. This results in an expected $6.02+2.01 N \mathrm{~dB} / \mathrm{bit}$ increase in SFDR.

Simulations using two noncommensurate tones confirmed a trend of roughly $10 \mathrm{~dB} / \mathrm{bit}$. For more than two tones, the simulation results become unreliable because the limited number of bins in a fast Fourier transform cause multiple nonnegligible intermodulation products to fall into the same frequency bin. Closer numerical evaluation of the effects of multitone quantization will be left for future research.

\section{EXAMPLE}

Consider a Flash ADC with differential-pair-based preamplifiers at the input, which amplifies the difference between the input signal and the different reference voltages generated through a resistor ladder. Assume that the input capacitance of each differential pair is $30 \mathrm{fF}$, and that there are no mismatches and offsets. The thermal noise generated by the ladder is equal to $\overline{v^{2}}=k T / C \approx 1.4 \cdot 10^{-7} \mathrm{~V}^{2}$, whereas the input-referred noise of the differential pair with drain resistors $R_{D}$ is equal to $\overline{v^{2}}=8 k T\left(2 / 3 g_{m}+1 / g_{m}^{2} R_{D}\right) \approx 10^{-17} \mathrm{~V}^{2}$ for $R_{D}=400 \Omega$ and $g_{m}=4 \mathrm{mS}$. The thermal noise generated by the reference ladder clearly dominates with $\sigma_{N}=\sqrt{1.4 \cdot 10^{-7}} \approx 0.38 \mathrm{mV}$.

The noise becomes important for the SFDR when $\sigma_{N}>$ $0.1 \mathrm{LSB}$, because then [using (14)], it contributes $1.7 \mathrm{~dB}$. Considering a realistic input voltage range of $500 \mathrm{mV}$, this will be the case for 7 bits, which gives an SFDR of 8.07 . $7+3.29+(0.38 / 3.9)^{2} \cdot 171.5=61.4 \mathrm{~dB}$. For 5 bits, the noise contribution is negligible, and the SFDR is found to be $43.7 \mathrm{~dB}$, whereas when using (1), the SNR will be $31.9 \mathrm{~dB}$. For 9 bits, the noise increases the SFDR by $28.0 \mathrm{~dB}$ to a total of $101.9 \mathrm{~dB}$.

\section{Conclusion}

The SFDR of a uniform quantizer, when quantizing a sinusoid without noise, increases by roughly $8 \mathrm{~dB} / \mathrm{bit}$, which was found by numerical evaluation of exact formulas and verified by simulations. This trend and the seemingly random deviations around it were mathematically explained. Adding noise decorrelates the quantization error from the input signal and, therefore, increases the SFDR. For Gaussian noise, the SFDR quadratically increases with the standard deviation of the noise in LSB. A numerical comparison between a simple approximation formula that has been introduced in this brief, and the exact value obtained from existing but complicated analytical formulas, shows an error of less then a few decibels, which is often acceptable for practical purposes. In the case of $N$ tones, the SFDR is expected to increase by $(6+2 N) \mathrm{dB} / \mathrm{bit}$, which was derived in the same way as for the single-tone situation.

These results can be applied to the design of systems without having to use the exact formulas. This can save a lot of time and effort. The results relate the SNR, the SFDR, and the number of quantization levels, and knowing two of them allows easy calculation of the third.

\section{ACKNOWLEDGMENT}

The authors would like to thank X. Gao for providing valuable references and $\mathrm{P}$. Veldhorst for his assistance with the practical example.

\section{REFERENCES}

[1] H. Pan and A. Abidi, "Spectral spurs due to quantization in Nyquist ADCs," IEEE Trans. Circuits Syst. I, Reg. Papers, vol. 51, no. 8, pp. 1422-1439, Aug. 2004.

[2] M. Wagdy and W. Ng, "Validity of uniform quantization error model for sinusoidal signals without and with dither," IEEE Trans. Instrum. Meas., vol. 38, no. 3, pp. 718-722, Jun. 1989.

[3] B. Le, T. Rondeau, J. Reed, and C. Bostian, "Analog-to-digital converters: A review of the past, present and future," IEEE Signal Process. Mag., vol. 22, no. 6, pp. 69-77, Nov. 2005.

[4] N. Blachman, "The intermodulation and distortion due to quantization of sinusoids," IEEE Trans. Acoust., Speech, Signal Process., vol. ASSP-33, no. 6, pp. 1417-1426, Dec. 1985.

[5] M. Abramowitz and I. Stegun, Handbook of Mathematical Functions With Formulas, Graphs and Mathematical Tables. New York: Dover, Jun. 1964.

[6] M. Wagdy, "Effect of various dither forms on quantization errors of ideal A/D converters," IEEE Trans. Instrum. Meas., vol. 38, no. 4, pp. 850-855, Aug. 1989.

[7] M. Keshner, "1/f noise," Proc. IEEE, vol. 70, no. 3, pp. 212-218, Mar. 1982.

[8] A. Kokkeler and A. Gunst, "Modeling correlation of quantized noise and periodic signals," IEEE Signal Process. Lett., vol. 11, no. 10, pp. 802-805, Oct. 2004. 